Chapter 4

\title{
Managing Pharmacy Operations with People and Technology
}

\author{
Margaret O. Afolabi and \\ Omoniyi Joseph Ola-Olorun \\ Additional information is available at the end of the chapter \\ http://dx.doi.org/10.5772/55253
}

\section{Introduction}

Operations are the processes by which people, capital, and materials (inputs) are combined to produce the services and goods consumed by the public (outputs). Essentially, operations add value to the final product over and above the product's cost. Operations Management (OM) is the functional area of business primarily devoted to the creation, planning, and management of the resource capabilities used by a firm to create products or services. Galloway (2000) viewed operations management as all activities concerned with the deliberate transformation of appropriate range of resources to produce the organisation's intended outputs. This transformational model may be represented as in Fig 1. Inputs are the resources such as personnel, capital, equipment, information and technology while outputs include the actual delivery of required goods and services.

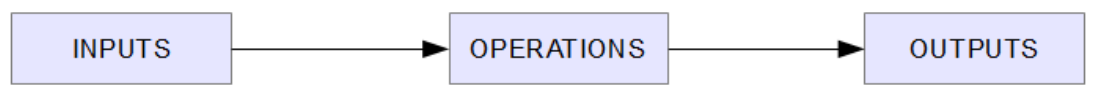

Figure 1. Transformational model of operations management

The resource capabilities comprise the inputs such as the work force (e.g., skills), technology (e.g., manufacturing equipment and information-based technology), and processes (e.g., supply chain, inventory-distribution system, quality control system, material flow system, production planning methods, monitoring system, etc.) all of which typically represent a significant portion of a firm's total costs and controllable assets. Since resource capabilities determine the types of products and services a firm can offer to the marketplace as well as the 
associated cost (price), quality attributes, and lead-times necessary to meet demand, the operations function is a critical driver of competitive advantage. Moreover, recent forces such as technology change and increased competition in cost, time and quality have elevated the distinctive competence that can be obtained from the effective management of operations function.

Healthcare operations management is considered as the quantitative management of supporting business systems and processes that transform resources (inputs) into health care services as outputs (Langerbeer II, 2008). Pharmacy operations are carried out within the healthcare system and have a mix of both intangible and tangible characteristics. Appropriate resources are transformed to create the pharmaceutical services which form intangible components of the operations. These services are knowledge - based and have high levels of customer interactions. The services accompany health commodities which are tangible products; the logistics and supply of which are major functions of operations management.

The objectives of the chapter are to

1. Describe the scope of operations management in health care

2. Justify the need for technology and automation in pharmacy operations

3. Highlight some types of technology employed in pharmacy operations

4. Examine human resource issues of operations and technology in the pharmacy

5. Highlight process workflow of prescription filling in a pharmacy

6. Describe process improvement approaches to optimise patient flow in a pharmacy.

\section{The scope of operations management in healthcare}

Operations management is the set of intrinsic or internal processes and decisions that help address costs, process, technology and productivity. There are obvious lapses in how the processes and systems of healthcare are managed hence the need for a greater focus on applying management science to improve the processes and outcomes. Quantitative management implies the use of analytical tools as well as extensive use of process and quality improvement techniques to drive improved results. Similarly, using technology to further automate and streamline some processes in healthcare operations can help reduce costs and maximise efficiencies. The scope and functions of operations management include strategies to reduce costs and variability, improving logistics flow, quality of customer service and productivity and continuously improving business processes.

Essentially, operations management of pharmacies is a discipline of management that integrates scientific or quantitative principles to determine the most efficient and optimal methods to support pharmaceutical services in patient care delivery. There must be an adoption of operations management techniques into pharmacy practice to help drive improvements and efficiencies. For instance, incorporating queuing theory and scheduling 
optimisation methods help to reduce wastes and cycle time in patient care. The scope of operations management of the pharmacy includes all functions related to management systems and business processes underlying the delivery of pharmaceutical services. This includes extensive focus on the process workflow, physical layout, capacity design, physical network optimisation, staffing functions, productivity management, supply chain and logistics, quality management and process engineering.

\section{Technology and automation in pharmacy operations}

Technology is an increasingly important element in operations management. It may be defined as "anything that replaces routine or repetitive tasks that were previously performed by people or which extends (or enhances) the capability of people to do their work" (Rough, 2001). Merriam-Webster (2009) defines technology as "the practical application of knowledge especially in a particular area" and "a capability given by the practical application of knowledge". On the other hand, automation refers to "any technology, device or machine that is linked to or controlled by a computer and used to actually do work that was previously done by humans' (Rough, 2001). In essence, all automation is technology, but the reverse is not necessarily true.

Automation is a trend in technological development, which seeks to eliminate direct manual involvement in control procedures; whereas, mechanisation is a component part of automation and it is concerned with replacement of manual labour with machine (Encyclopaedia Britannica, 2010). The use of technology and automation is paramount in the focus of operations management on improvements of business support services. Technology as an enabling factor has an indirect impact on work and it should be considered whenever quality and efficiency is low, particularly in processes such as dispensing of medicines in a pharmacy. These activities are repetitive in nature but amenable to some basic technology. The decision to substitute technology for labour in such support services is the only way to reduce processing and transactional costs over the long run.

The Local Enterprise Authority (2009) identified three types of technology; these are product, process and support technologies. Product technology is embodied in the organisation's product and it is often an important element of the product. It provides the product's innovative features, improved performance, and the very materials that goods are made from. Scientific knowledge is applied in development and production of medicines by pharmaceutical manufacturers and other healthcare researchers for the purpose of improving health and wellbeing. For this reason, medicines and other pharmaceutical products can be regarded as technology. Examples are drug delivery systems such as transdermal patches and implantable computer incorporated drug administration devices. These are classified as product technologies covered in the area of pharmaceutical technology (Bozzette et al., 2001).

Process technology is employed in the production process and refers to the actual method used to transform inputs into outputs or finished goods and services. It concerns the equipment used, the operations performed on materials or customers in the service systems and such 
technology could be manual, automatic or mental. In most cases, a range of technologies is available for defining a specific process and each one has advantages and disadvantages, which must be weighed in the light of a firm's competitive priorities. Technology is also often a crucial part of the process to produce and/or deliver the product, for example digital technology to produce and deliver published medicine information materials for use in patient care. On the other hand, support technologies are used to perform certain other activities that are not embodied in the product or production process of an organisation. Among such technologies are information and communication tools such as software packages, computer networks, and quality assessment technology.

The goal of operations management is effective and efficient use of resources which is enabled by technology; hence its need in the management of pharmacy operations. The benefits of technology to support pharmacy operations include improvement in safety, efficacy and economy of medicines (Slee et al., 2002). The compelling need for technology and automation is partly the result of innovations in creation and design of new technologies and increased labour costs which call for more cost effective production systems and operations (Garsombke and Garsombke, 1989). Developments in technology can be seen as developments in the innovation process and technological innovation is the first commercial introduction of a new technology, which may take the form of a product, process or service (Ilori, 2006).

The use of technology in pharmacy operations improves medication safety in patient care and also enhances efficiency of the medical process. Furthermore, it improves the documentation of care (Kelly, 2006). Nevertheless, it is believed that the application of sophisticated technology in operations management poses its own challenge; while technology improves medication safety and processes in terms of reduced medication errors, there is little evidence to suggest that any single technology has the potential to radically reduce adverse events. However, there are incremental benefits to be derived from each and full benefits may only be realised when several technologies are used and integrated (Slee et al., 2002).

\section{Some types of technology employed in pharmacy operations}

Various types of technology have found application in pharmacy operations. They can be grouped into mechanisation technologies, quality assessment technology, information and communications technologies (ICTs), automation technologies and the newly evolving biotechnology

\subsection{Mechanisation technologies}

Mechanisation has been described as the use of machines, either wholly or in part, to replace human or animal labour (Encyclopaedia Britannica, 2010). It serves as a means of providing human operators with machinery to assist them with the physical requirements of work. Example of mechanisation in pharmacy operations within the hospital is the use of electrical mixing vessel in compounding medicines. The technology for compounding medicines 
include facilities for making individualised doses of medicines such as intravenous feeding solution for patients unable to eat, or doses of anticancer chemotherapy.

Compounding in pharmacy operations can be described as the creation of a drug product by mixing ingredients (Jeffcoat, 2004). Sometimes the needs of a patient cannot be met by a licensed medicine as in the case of a child for whom a particular medicine is not commercially available as a liquid. In such circumstances, suitable products that are required to meet the same high standard as those of pharmaceutical industry can be prepared in the hospital pharmacy.

The tools and equipment used for compounding are simple laboratory equipments such as mortar and pestle, glassware and simple machines. Similarly, tablet counting for pre-packaging, repackaging and dispensing is one of the routine tasks in the hospital pharmacy. The traditional technology adopted has been the counting tray but the tablet counting machine (or tablet counter) is now available.

\subsection{Quality assessment technology}

Quality assessment technology is used for the task of assessing product quality. The technology used to assess the quality of drug products spans a wide spectrum of technological artifacts, some of which are chemical reagents, chemical and biochemical equipment and instruments as well as high precision instruments for pharmaceutical analysis. However the main thrust of quality assessment of drug products is chemical analysis.

Counterfeiting of pharmaceutical products has assumed a global concern and one of the measures for checking this menace is the use of low cost mobile quality assessment technology. The GPHF-Minilab ${ }^{\circledR}$ is a form of mobile quality assessment technology for detecting counterfeit and substandard quality medicines (Jahnke et al., 2001; Jähnke, 2004). The equipment is a portable, tropics-compatible and easy-to-use mini laboratory, developed mainly for use in developing countries, which are particularly affected by counterfeit drugs.

The GPHF-Minilab ${ }^{\circledR}$ is designed so that all the devices necessary for testing the drugs can be accommodated in two portable units, each about the size of a suitcase and the GPHF-Mini$\mathrm{lab}^{\circledR}$ can be used without an external energy source. The mini-laboratory contains, in addition to the reference substances for the targeted active ingredients, all the necessary laboratory appliances such as test tubes, pipettes, pre-coated TLC plates and developing chambers as well as battery-powered UV lamps set to different wavelengths and the instructions and necessary solvents and reagents for the tests.

\subsection{Information and communications technologies (ICTs)}

Information and communications technology (ICT) has been described as consisting basically of information handling tools which include a varied set of goods, application and services that are used to produce, store, process, distribute and exchange information (Hamelink, 1997). It is an umbrella term that includes all technologies for the manipulation and communication of information such as radio, television, cellular phones, computer network and satellite systems as well as the various services and applications associated with them. 
Hamelink (1997) described ICTs as those technologies that enable the handling of information and facilitate different forms of communication which include capturing technologies (e.g. camcorders), storage technologies (e.g. CD - ROMS), processing technologies (e.g. application software), communication technologies (e.g. Local Area Networks) and display technologies (e.g. computer monitors).

The overall ICT infrastructure comprises the computer and communication technologies and the shareable technical platforms and databases (Ross et al., 1996). Most of the technologies adopted in pharmacy operations are ICT-based technologies. The key ICTbased technologies to be discussed include the computer, other computer-based technologies and the telephone.

There has been earlier report that differentiated between telepharmacy and e-Pharmacy (Sood et al., 2008). Telepharmacy connotes the delivery of pharmaceutical to clients in a remote location. It is not a technology but a method which employs technology whereas e-Pharmacy is an innovative concept associated with electronic transactions, e-prescription systems, decision-support systems, among others, in the delivery of pharmaceutical services. In epharmacy, the pharmacist provides professional services to clients by electronic means, usually an ICT (e.g., telephone, internet, etc.). Peterson (2010) described telepharmacy as a branch of pharmacy practice that allows pharmacists to provide pharmaceutical care to patients at a distance through the use of the state-of-the-art telecommunications technology which allows a licensed pharmacist to supervise telepharmacy site through the use of video conferencing technology.

\subsubsection{Computer applications in pharmacy}

The computer is an electronic device that is capable of accepting data in a prescribed form, and processing and supplying the results in a specified format as information or signal to control automatically some machine or process (Graf, 1999). It is extremely fast, accurate and useful as an information processing device. In the contemporary world, the adoption of computers is integral to technological advances in any operational system.

The versatility of the computer for processing and communication of data has made it useful in pharmacy operations. The computer software is the adaptable part of the computer system but the proficiency of a user in handling the computer is a major factor in its usefulness. There is a minimum level of capability required for the successful use of a computer and this can influence the acceptance or otherwise by a potential user (Dixon and Dixon, 1994). The application could be home-developed or enterprise package and it could be stand-alone or integrated with other applications or technologies. According to Kling (1996), the relevant factors in the effect of technology, particularly computerisation at the workplace, pertain to gender equity, control, support systems, social design and new ways of organising work; but no Single logic has been applied towards changing the work system with computerisation. Computerisation has found immense application in pharmacy operations because of the benefits of enhanced efficiency and effectiveness of the work system. Staff efficiency becomes evident as more time is freed from routine tasks (Afolabi, 2005). Such available time may then be spent on patient counselling and other clinical functions to reduce potential medication 
errors for better health outcome. The computer system has been found to be the most widely adopted ICT in pharmacy operations in the hospitals (Perri et al., 1988; Santell, 1995 and Quick et al., 1997). The system can operate at the departmental level, at the hospital level or even networked with the world wide web. The computer-based technologies currently employed in pharmacy operations include information support applications, decision support applications, bar-code technologies, the personal digital assistants (PDAs) and other hand-held devices.

\subsubsection{Computer-based technologies}

\subsubsection{Information support applications}

Clinical informations systems are those that are integrated through the whole hospital system as hospital information system such as electronic health record or its variant, the electronic medical record, designed to capture the all clinical and administrative information about a patient and has come to be the main tool of any clinical information system. The record contains patient demographics, medical history, previous admission information, previous surgery information, and obstetric history. Computerized provider order entry (CPOE) refers to any system in which clinicians directly enter medication orders (and, increasingly, tests and procedures) into a computer system, which then transmits the order directly to the pharmacy (Grizzle et al., 2007). Electronic prescribing or e-prescribing involves a process of using a computer to enter, modify, review and output or communicate prescriptions electronically to a patient's pharmacy (ASHP, 2007). It is used for sending an accurate, error-free and understandable prescription directly to a pharmacy from the point-of-care and further enhances the quality of care and patient safety by integrating medication order into the overall process of medical care delivery (Thompson and Brailer, 2006).

The pharmacy information system could operate within the hospital for managing inpatients or linked with other healthcare facilities such as community pharmacies for outpatients. An example is the Pharmacy Care Plan which is a computer based form used for keeping patients record for the purpose of pharmaceutical care (Winslade et al., 1996). It integrates data from various other systems within the hospital to improve therapy, safety or costs.

\subsubsection{Decision support applications in pharmacy operations}

Decision support applications can be classified into clinical decision support systems (CDSS) and Clinical Pharmacokinetics Computer Programs (CPCP).

Clinical decision support systems (CDSS or CDS) are interactive computer programs, which are designed to assist physicians and other health professionals with decision making tasks. They provide clinicians with patient-specific assessments or recommendations to aid clinical decision making (Kawamoto et al., 2005). The methodology of using CDSS forces the clinician to interact with the CDSS utilising both the clinician's knowledge and the CDSS to make a better analysis of the patient's data than either human or CDSS could make on their own. 
Clinical pharmacokinetics computer programs are computer software for use by pharmacists in analysing serum concentration data. They are employed for patients to individualise dosage regimens of highly toxic medicines and those with narrow therapeutic windows.

\subsubsection{Bar-code technologies}

A bar-code is an optical machine-readable representation of data (Okon, 2005). It is usually a series of vertical bars and spaces. The bars and spaces represent digitally encoded information which is designated by the width of each bar and space. The height of a bar code is irrelevant although it must be sufficient in size to allow an operator to easily read each bar with a scanning device (Okon, 2005). Since the codes are digitally made they can only be read and interpreted by means of electronic machines designed for this purpose hence bar-codes are normally read by optical scanners called bar-code readers, or scanned from an image by special software (Okon, 2005).

Bar-code technologies are applied in two ways either as Bar-code-assisted dispensing (Bar-D) or bar-code at the point of medication administration system (BCMA). They are designed to prevent medication errors in hospitals (Sakowski, 2005). They work with hardware that consists of a handheld scanning device connected to a wireless mobile computer. This mobile hardware communicates with other information equipment in the system through a server to record transactions. Some systems utilise a bar-code scanner-enabled personal digital assistant (PDA) to scan, confirm and store medication transaction information.

\subsubsection{Personal Digital Assistants (PDAs) and other hand-held devices}

These are small mobile computers such as the blackberry, apple iPhone and "smart phones" (Anderson et al., 2010). They usually have integrated ICT applications such as telephone, camera, scanner, etc. Their ability to handle vast amount of information and instantaneous speed of operation including switching on and off makes them vital for medicine information services.

\subsubsection{The use of telephone in pharmacy operations}

The Short Message Service (SMS) on mobile phones, as a form of e-messaging has been employed in promoting adherence to medicine therapy especially in the treatment of chronic diseases such as hypertension, HIV/AIDS, diabetes and osteoarthritis. Adherence is the degree to which patients conform to a given treatment plan or the extent to which patients take their medications as prescribed by healthcare providers. A review of published studies reported that up to $10 \%$ of hospital admissions are related to non-adherence.

One of the important aspects of pharmaceutical care is the provision of medicine information service (MIS) to patients. Mobile telephones are readily available and this form of technology can be used to communicate medicine information to patients with chronic ailments. 


\subsection{Automation technologies in pharmacy operations}

A number of technologies have been used to automate various aspects of pharmacy operations. Their aim is usually to replace manual labour and promote safety and economy. The four main benefits that result from the use of automation technologies in pharmacy operations include reduction in errors (Slee et al., 2002), improved use of space and workflow (Cairns, 2002) improved stock control and stock management (Martin, 2002) and improved departmental efficiency (Fitzpatrick et al., 2005) which reduces patient waiting times and allows pharmacists to use their time more effectively in pharmaceutical care.

Basically, there are three types of automated devices namely automated compounding and/or counting devices, automated medication supply systems and automated checking devices (Slee et al., 2002; Fitzpatrick et al., 2005). They could be for inpatient or outpatient services in the hospital (Anderson et al., 2010).

\subsubsection{In-patient pharmacy automation technologies}

The available inpatient pharmacy automation technologies include the automated checking device, smart infusion pumps, automated dispensing cabinets (ADCs) and robots (Anderson et al., 2010).

The automated checking device is a fully automated device which confirms, after a drug is prepared for distribution but prior to delivery to the patient, that the right drug has been labelled correctly for the right patient using technology such as bar-coding (Fitzpatrick et al., 2005).

\section{Smart pumps}

The "smart" infusion pump is a medication administration equipment for providing medication infusion at a specified rate. It has a computer "brain" with a database of standard intravenous (IV) preparations from which the required item can be selected. It can trigger alert when any of the limits set for it concerning dosage or other data has been exceeded.

\section{Automated dispensing cabinets}

Automated dispensing cabinets (ADCs) are computerised packaging equipment in which a consignment of medicines in unit dose packaging (as in a ward stock) are contained in locked drawers. The machine is able to dispense medications electronically in a controlled fashion and track medication use. The storing and picking of products and the labelling stage of the dispensing process are automated (Slee et al., 2007). ADCs dispense the required medicines and record the identity of the user, the patient and the medicines dispensed.

\section{Robots}

The pharmacy robots add kinetic components that mimic human activity to the capability of the ADCs by way of preparing, dispensing, and distributing medicines to various locations in the hospital. It employs the bar-coding technology for identifications by labelling or scanning as necessary. 


\subsubsection{Outpatient pharmacy automation technologies}

\subsubsection{Automated drug dispensing machines}

Automated drug dispensing machine (ADDM) also called automated compounding or counting device is an automated device that compounds, measures, counts, and/or packages a specified quantity of dosage units of a designated drug product (Fitzpatrick et al., 2005). The ADDM is interfaced with the pharmacy information system and it can fill, label and deliver prescriptions received from the system (Anderson et al., 2010).

\subsubsection{Automated kiosks}

Automated kiosks are devices that operate like Automated Teller Machines holding the medication that has been filled by the pharmacist and delivering the medicines to patients at their convenient time (Anderson et al., 2010). They allow for payment at the point of receipt.

\subsection{Biotechnologies in pharmacy operations}

The study of genome, the entirety of an organism's hereditary information, has led to the discovery of the sequence of genes in the human DNA (Ridley, 2006; Anderson et al., 2010). The DNA is the means by which genetic traits are transferred and the structure of each organism's DNA sequence determines the genotype and hence the characteristics of the organism. This knowledge has a great potential for applications in producing medicines that are tailored to each person's unique organic characteristics as well as to selectively target pathogens in disease management. The full benefit of biotechnology in pharmacy practice is still potential although its magnitude probably cannot be imagined for now.

\section{Human resource issues, technology and automation of work}

The workforce of an organisation, though viewed as part of the resources, actually forms the interface between the customers and the organisation and is significant in the management of operations. In fact, the workforce represents the organisation to the customer and is responsible for the system to be functional. It is necessary therefore to temper the technical focus of traditional operations management with the reality of human behaviour and employee satisfaction (New, 1998).

Automation as a trend in technological development is an enabling factor in the work system and should be considered in quality improvement strategies of operations management. The direct impact of automation could be felt at the micro level of a work system as this technological change can alter the nature of the tasks, work cycles, skill requirements, and worker interactions. With increasing automation, jobs tend to become more demanding, varied, interesting and challenging for many workers; although in some cases, such changes may be of a temporary nature. Technical know-how tends to become more 
important and workers may expect increased job content together with increased demands on skills, knowledge and training.

Automated systems may result in greater complexity and responsibility, and therefore greater intrinsic rewards, but often at the expense of worker inactivity. Furthermore, automation has considerable effects on social interactions. The greater distance between workers caused by automated systems may result in reduced social relationship, and also, there is an increased separation of workers from both operations and their outputs. On the other hand, in automation there could be an increased contact between workers and their supervisors leading to improved worker - supervisor relations. Similarly, increased training needs are often associated with the wider responsibilities of automated jobs.

Occasionally, increased stress may be experienced in work situations approaching full automation. This may be as a result of emphasis on vigilance and monitoring duties. The importance of minimising process disruption, the consequences of breakdowns and comparative inactivity of workers also contribute to stress conditions in automation.

One reason for the automation of work is the desire to remove workers from unsatisfactory working environments. Such criteria for automation include risk of accident, monotony of work, excessive physical stress and bad working conditions (Wild, 1999). It may be necessary, therefore, to have some means to check the acceptability or appropriateness of working conditions. This will enable existing work systems to be appraised in order to establish whether there is the need for a change and similarly a new work system design can be checked for acceptability or appropriateness by the workers before implementation.

Although automated systems can contribute to an organisation's complexity, they can also help the organisation to cope with such complexity. The speed and accuracy of the computer, as an automated technique, seem attractive for decentralised decision making while top management has time for planning and innovation. Computer - based information could improve the managers' capabilities making them better employees and more important to the success of the organisation. Some routine, clerical activities are easily adaptable to computerisation, and procedures may be developed to handle structured repetitive tasks. For instance, in a pharmacy, such repetitive tasks as prescription billing, documentation, and stock control functions may be taken over by the computer (Slee et al., 2002). In these situations, the work is repetitive and can be described in a clear procedure for routine transaction processing. With this development much of the mundane, tedious routine jobs of prescription processing may be automated while the unstructured, non routine and more skilled decisions can then be handled by the pharmacists.

In clinical functions, creativity, insight and professional judgment are needed on the part of the pharmacist who has to advice on choice of medication in a patient's therapeutic plan. Much of the problem solving activities in these areas require knowledge of the medicines and their therapeutic efficacy. Assistance from computer - based information systems helps management decision making and this could improve a pharmacist's advisory skill in patient care. Thus, rather than replace or displace pharmacists' ranks, introduction of automated techniques, such as automated medicine information systems, should create a demand for more 
highly qualified and better-trained pharmacists. Computer training and enhanced clinical skills should therefore be an integral part of pharmacy education and retraining programs. The workers in an organisation should be treated as a major long term investment with enhanced skills in order that the company may realise its full potentials and in such a way that these employees may feel appreciated (Scaborough and Zimmerer, 2000).

Much as technology is desirable to enhance operational effectiveness, the implementation of new technology will benefit from due attention to factors connected to employee perceptions and acceptance of the change. Genus and Kaplani (2000) examined the need to consider the behaviour of employees in connection with operations and changes in the design of the work system brought about by technology. He concluded that shared values between an organisation and the work force would facilitate the implementation of operational innovations. Similarly, Savery and Choy (1999) opined that a strategic choice of technology should include due attention to human resource management issues and a consideration of the assets and appropriate facilities for operational effectiveness. Therefore, for an organization to realise its full potentials, the workforce should be motivated with a conducive and friendly work environment.

It has been shown that content and design of the work system can also be a source of motivation or otherwise for workers (Scaborough and Zimmerer, 2000). A lack of employee motivation and the absence of shared values could pose as barriers to employee involvement in and commitment to continuous improvement objectives in an organisation (Afolabi and Oyebisi, 2007a). Appropriate staff training and a high degree of motivation may achieve the harmony of staff perception and organisational intention in the adoption of technology to enhance performance.

The pharmacy unit is a service operating system and it is expected to provide relevant and adequate infrastructure and tools required to satisfy client needs in terms of appropriate medicine supply and information services as well as facilitate social and appropriate professional interactions in the health system. In addition, the system should enhance adequate patient flow.

\section{Operations management and patient flow in hospital pharmacies}

Patient flow optimisation opportunities occur in many healthcare settings especially at the pharmacy units of hospitals in resource limited countries; where patients wait in lines to fill their prescriptions. There could be many causes of poor patient flow in such circumstances but the major cause is variability of scheduled demand. Variability is the inconsistency or dispersion of inputs and outputs and this threatens processes because it results in uncertainty. For instance, if there are 50 patients typically seeking care at the outpatient pharmacy unit within a certain time period and 100 appear the following period, it becomes difficult for staff to control waiting times and to manage patient flows. Improving flow means seeking higher throughput or yields for the same level of resource input. 
Throughput is the rate or velocity at which services are performed or goods are delivered. If patient volumes double but a hospital maintains the same historical inventory levels of pharmaceutical supplies, this represents significant improvements in material flow, because assets have higher utilisation and turns. Staffing and resource consumption should be tied directly with patient volumes and workload; if patient volume increases, the resources should also increase.

Operations analysis helps to track demand variability which is consequent on a surge in patient demand. Managing this variability allows a change in staffing mix and scheduling to accommodate the changes in demand. Staffing at the peaks will cause excessive costs while staffing for the valleys or low points will cause long lines periodically due to limited resources and therefore service quality issues; on the other hand, staffing for the average demand is the most common but suboptimal approach. Managing patient flow is an operating issue in a healthcare organisation but effective and timely management provides an opportunity for desired outcome. Significant opportunities exist to improve capacity and reduce costs by improving patient flow using the formal methods of process improvement.

\section{Process improvement approaches to optimise patient flow in a hospital pharmacy}

Filling of prescriptions forms the core of pharmacists' activities in the hospitals in Nigeria. However, considerable delays were observed in dispensing operations in the pharmacies and these delays result in long patient queues, particularly at peak periods. A process improvement project was carried out to examine dispensing procedure at the pharmacy unit of OAUTHC, and to identify aspects of the process design (task elements) that contributed to patient delays.

Data were collected by direct observation of dispensing workflow and a time study of the procedural elements. A checklist was used for a systematic recording of the various activities and queuing models were used to characterise the waiting line structures at the pharmacies with a view to simulating optimal utilisation factors $(\mathrm{Pw})$ for the service channels. Time elements of dispensing operations revealed extensive delay components which contributed to excessive patient waiting time.

The operational problems identified in the work process were due to tortuous procedure for prescription filling and the volume of transactions at the cashier stand. The operating characteristics of the paying systems were not optimal with only one service channel. The delays observed were substantially due to existing work procedure in the pharmacy and the volume of manual transactions which are amenable to newer technologies. Some of the dispensing task components were not essential and these elements could be removed or merged in order to reduce patient waiting time (Table 2). The details of the project are presented in section 7.2. 


\subsection{Process maps}

Extended waiting time to fill prescriptions in hospital pharmacies can be addressed by using some process, improvement tools. A process map or flowchart is a graphic depiction of a process showing inputs, outputs and steps in the process. Figure 2 shows a process map that illustrates the activities involved in prescription filling at the pharmacy unit of a university teaching hospital in Nigeria. Figures $3 a$ and $3 b$ present the staff process chart, indicating the tasks and delay components of the operating system.

Typically, process maps are used to understand and optimise a process and the process may be charted from the viewpoint of the material, information, the worker carrying out the work or the customer being processed. In Figure 2, the customer being processed is the prescriptioncarrying patient within the healthcare. Process mapping is a basic quality tool and an integral part of most improvement initiatives. The steps for creating the process map include an observation of the process and a description to ensure the real activities are captured. Boundaries are determined for the activities and the process tasks and subtasks are listed and arranged in order as a written procedure or protocol (Table 1). A formal flow chart is then generated using standard symbols for process mapping. This is then checked for accuracy and additional data on process performance may be added depending on the purpose of the flowchart. Service blueprinting is another quality improvement tool and a special form of process mapping as shown in figure 4 . The process is mapped from the point of view of the customer.

Basically, the purpose of a service blueprint is to identify points where the service might fail to satisfy the customer and then redesign or add controls to the system to reduce or eliminate the possibility of failure. Three distinct actions are delineated in serve blueprinting and these are the customer actions which show the nature of customer involvement in the process and the interactions, onstage actions which are visible to the customer, the backstage and support processes which are not visible to the customer. A service blue print specifies the line of interaction, where the customer and service provider come together, and the line of visibility, that is, what the customer sees or experiences, the tangible evidence that influences perceptions of the quality of service.

Process maps provide a visual representation of the process and this offers an opportunity for improvement through inspection. The maps allow for branching in a process and provide the ability to assign and measure the resources in each task. Process mapping are the basis for modelling the process via computer simulation software or using other process technology. Simulation is a modelling technique that may be used to evaluate the effects of possible changes on an operating system. The process of simulation consists of model development, model validation and an analysis of the output to optimise a process or manage risk. Discrete event simulation using the queuing theory may be used to model system flows as an improvement strategy. The following section (Section 7.2) applies these concepts to process improvement in a hospital pharmacy with emphasis on patient waiting time. 


\subsection{Operations management project : The case of OAUTHC pharmacy}

In order to demonstrate possible application of the process improvement tools described previously, a patient- flow process improvement project at the pharmacy unit of a teaching hospital was examined.

It was observed that outpatients experience considerable delays to fill prescriptions at the pharmacy unit of a university teaching hospital ( OAUTHC) and this was identified as an important area on which to focus improvement efforts. The goal of the project was to reduce patient waiting time while optimising capacity utilisation of the pharmacy resources in the hospital.

The first step for the project team was to identify the need to facilitate patient flow with improved dispensing operations at the pharmacy. The specific tasks in the project were:

1. Observe dispensing operations and write out the detailed steps using a checklist (Table 1)

2. Develop a flow chart of dispensing to outpatients (Figure 2)

3. Measure specific metrics for the procedural elements (Table 1) taking an average of observations for one week

4. With the flow chart and specific metrics, identify the delay components of the operating system(Figure 3 \&Table 2)

5. Use simple process improvement techniques to make changes in the process, then measure the results

6. Collect data needed to build a realistic simulation model based on queuing theory (Tables 3 \& 4)

7. Develop the simulation model and validate it against real data

8. Use the simulation model to conduct virtual experiments to improve patient flow.

9. Implement promising improvements and measure the results of the changes.

\footnotetext{
A. Initial patient contact and information collection

1. Clerk receives prescription and takes prescription to the pharmacist for pricing and processing

2. Clerk gives the prescription to patient
} 


\section{B. Payment time}

1. Patient arrives at the queue

2. Patient gives prescription to the accounts clerk

3. Patient receives the prescription from the clerk and pays for medication

\section{Submission of prescription and dispensing}

1. Clerk receives prescription from the patient after payment and takes the prescription to the pharmacist for processing

2. Pharmacist receives prescription from the clerk and edits the form

Pharmacist telephones physician for refill authorisation

Pharmacist queries any inappropriate prescribing

\section{Obtain and package medication}

1. Pharmacist writes and affixes label to the container

Attendant brings the medication and instructs patient

Pharmacist counsels patient (occasionally)

Pharmacist gives medication

\section{E. Final clerical processing and cleaning \\ 1. Pharmacist files patient prescription \\ 2. Attendant performs clean- up}

Table 1. Procedural elements of outpatient dispensing in the pharmacy 


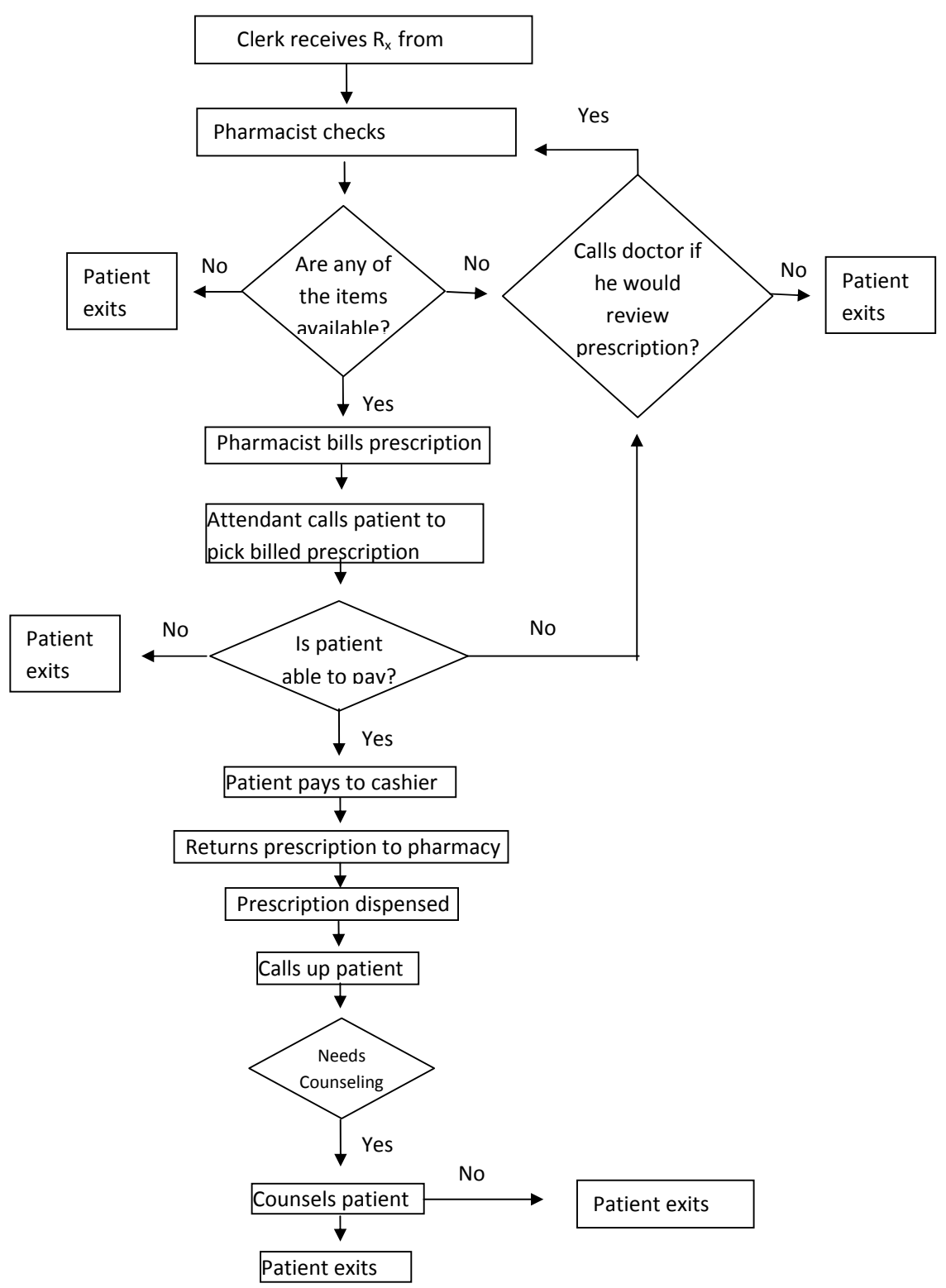

Figure 2. Process flow chart for prescription filling to outpatients 
Patient arrives and clerk receives prescription

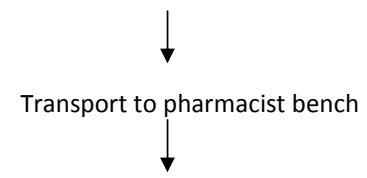

Pharmacist checks

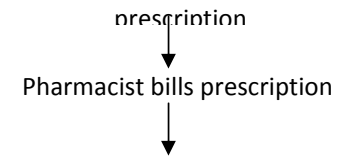

Pharmacist calls clerk to pick prescription<smiles>[3H][3H]</smiles>

Clerk calls patient to pick prescription

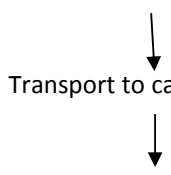

Transport to clerk's counter Transport to Pharmacist bench

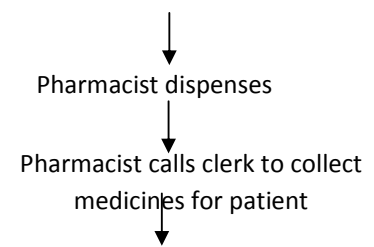

Clerk calls patient to collect drugs

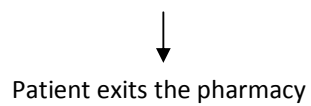

$\mathbf{a}$

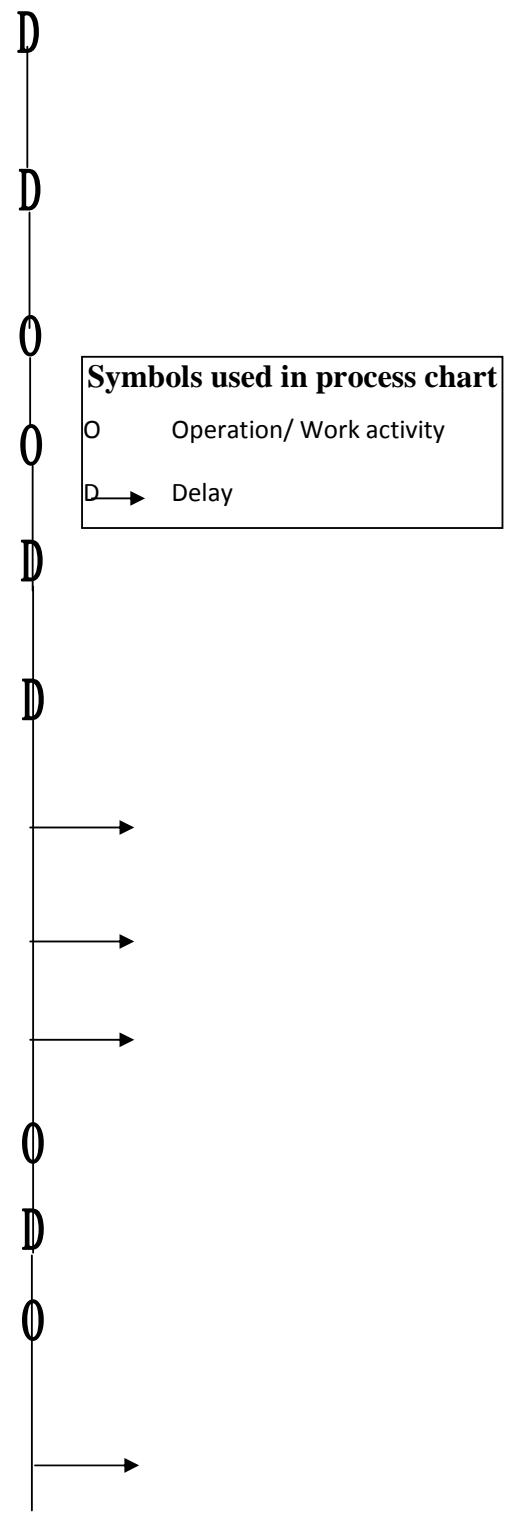

b

Figure 3. a: Staff process chart for dispensing to outpatients; b: Delay and process chart for dispensing to outpatients 
Time spent ( $\mathrm{min}$.)

Components of dispensing procedure

\begin{tabular}{ll}
\hline Processing Delay \\
\hline
\end{tabular}

\begin{tabular}{|c|c|c|c|c|}
\hline & & & & \\
\hline & Mean & $\%$ & Mean & $\%$ \\
\hline Patient waits for clerk to collect prescription & * & * & 0.76 & 4.45 \\
\hline Clerk takes prescription for billing & 0.15 & 0.88 & * & * \\
\hline Prescription placed on dispensing table for billing & * & * & 0.44 & 2.57 \\
\hline Pharmacist bills and reviews prescription & 0.62 & 3.63 & * & * \\
\hline Billed prescription placed on dispensing table for clerk & * & * & 0.60 & 3.51 \\
\hline Clerk takes prescription to patient & 0.18 & 1.05 & * & * \\
\hline Patient takes prescription to cashier & 0.14 & 0.82 & * & * \\
\hline Patient waits on queue for payment & * & * & 8.68 & 50.79 \\
\hline Cashier collects money, issues receipt and records & 1.42 & 8.31 & * & * \\
\hline $\begin{array}{l}\text { Patient collects receipt and prescription; and takes them to the } \\
\text { clerk }\end{array}$ & 0.18 & 1.05 & * & * \\
\hline Patient waits for the clerk to collect receipt and prescription & * & * & 0.56 & 3.28 \\
\hline Clerk takes prescription and receipt to pharmacist & 0.12 & 0.70 & * & * \\
\hline $\begin{array}{l}\text { Prescription and receipt placed on dispensing table for } \\
\text { pharmacist to fill }\end{array}$ & * & * & 0.58 & 3.39 \\
\hline Pharmacist fills prescription & 1.38 & 8.07 & * & * \\
\hline Medicine placed on dispensing table for clerk & * & * & 0.86 & 5.03 \\
\hline Clerk takes medicine to patient, instructs and dispenses & 0.42 & 2.46 & * & * \\
\hline Total & 4.29 & 25.10 & 12.80 & 74.90 \\
\hline
\end{tabular}

Total patient waiting time $=17.09$ min. ${ }^{*}=$ Nil

Table 2. Observed time distribution between processing and delay components for each task of the dispensing process at the pharmacy. 


\begin{tabular}{|c|c|c|c|c|c|c|c|c|c|}
\hline \multirow{2}{*}{ Hospital } & \multicolumn{9}{|c|}{ Waiting line parameters } \\
\hline & No of Channels & $\lambda / \min$ & $\mu / \min$ & Po & $\mathrm{n}_{\mathrm{s}}$ & $\mathrm{n}_{\mathrm{q}}$ & $t_{s}(\min )$ & $t_{q}(\min )$ & $\mathbf{P}_{\mathrm{w}}$ \\
\hline \multirow{3}{*}{ OAUTHC } & 4 & 0.62 & 0.40 & 0.20 & 1.60 & 0.05 & 2.58 & 0.08 & 0.08 \\
\hline & 3 & 0.62 & 0.40 & 0.20 & 1.82 & 0.27 & 2.94 & 0.44 & 0.25 \\
\hline & 2 & 0.62 & 0.40 & 0.12 & 3.83 & 2.28 & 6.18 & 3.68 & 0.66 \\
\hline
\end{tabular}

Where:

$\lambda=$ Arrival rate

$\mu=$ Service rate

$P_{o}=$ The probability of no client in the queue system

$n_{s}=$ The average number of clients in the queue system

$\mathrm{n}_{\mathrm{q}}=$ The average number of clients in the queue waiting for service

$t_{s}=$ The average time a client spends in the queue system (waiting time + service time)

$t_{q}=$ The average time a client spends in the queue waiting for service

$\mathrm{P}_{\mathrm{w}}=$ The probability that an arriving client has to wait for service

(utilisation factor or capacity utilisation of the facility)

Table 3. Operating characteristics of patient waiting lines in the pharmacy

\begin{tabular}{|c|c|c|c|c|c|c|c|c|c|}
\hline \multirow{2}{*}{ Hospital } & \multicolumn{9}{|c|}{ Waiting line parameters } \\
\hline & $\lambda / \min$. & Models & $\mu / \min$. & $\mathrm{n}_{\mathrm{s}}$ & $\mathrm{n}_{\mathrm{q}}$ & $t_{s}(\min )$ & $\left.t_{q(} \min \right)$ & $\mathbf{P}_{\mathrm{w}}$ & $\mathbf{P}_{0}$ \\
\hline \multirow{3}{*}{ OAUTHC } & 0.60 & A & 0.67 & 8.6 & 7.68 & 14.30 & 12.80 & 0.90 & 0.10 \\
\hline & 0.60 & B & 0.86 & 2.31 & 1.64 & 3.85 & 2.73 & 0.70 & 0.30 \\
\hline & 0.60 & C & 0.92 & 1.88 & 1.24 & 3.13 & 2.07 & 0.65 & 0.35 \\
\hline
\end{tabular}

Where:

$\lambda=$ Arrival rate

$\mu=$ Service rate

$\mathrm{P}_{\mathrm{o}}=$ The probability of no client in the queue system

$\mathrm{n}_{\mathrm{s}}=$ The average number of clients in the queue system

$\mathrm{n}_{\mathrm{q}}=$ The average number of clients in the queue waiting for service

$t_{s}=$ The average time a client spends in the queue system (waiting time + service time)

$t_{q}=$ The average time a client spends in the queue waiting for service

$\mathrm{P}_{\mathrm{w}}=$ The probability that an arriving client has to wait for service

(utilisation factor or capacity utilisation of the facility)

Table 4. Operating characteristics of one cashier service channel with varied service rates 


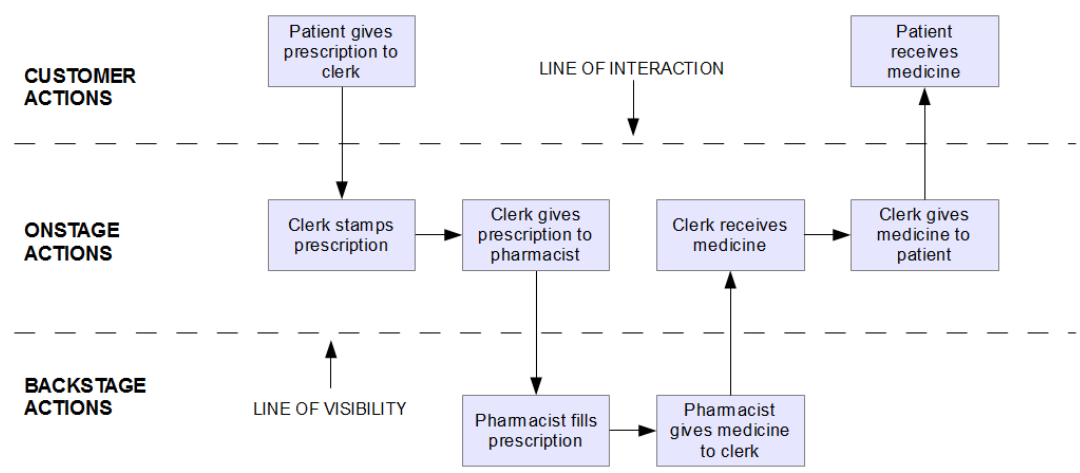

Figure 4. Service blueprint of prescription filling in a hospital pharmacy

\subsection{Operating characteristics of outpatient waiting lines at OAUTHC pharmacy using the simulation model}

A patient's experience of waiting can radically influence his/her perceptions of service quality and various studies have been carried out on queues in pharmacy systems and strategies to promote customer satisfaction (Lin et al.,1996; Lin et al., 1999). Simulation studies and queuing theory had been used to explore how changes in patient arrival rates and the time taken to dispense prescriptions were likely to affect patient waiting times (Toohey et al., 1982, Afolabi and Erhun, 2003). Some of the authors used simulation model to demonstrate that waiting times could be reduced by staggering pharmacists' break periods. Simulated analysis of patient queues in other studies examined the relationship between staff organisation, patient arrivals and waiting times in hospital pharmacies (Slowiak et al., 2008).

Queuing models give descriptions of queuing systems and such descriptions provide valuable inputs to the decision-making process of an operating system. For instance, given a queuing model, the descriptions of a queuing system that may be obtained include the probability that there will be a number of clients waiting in the queue and the probability of the systems being idle. Similarly the description gives an idea of the average number of clients waiting in the queue and the expected number of clients in the system (i.e. queue plus service facilities). The time estimates may also be obtained from the description as the model gives the probability of the service times, the average time a client spends on the waiting line and the total time required to get through the entire queuing system (Panthong and Daosodsai 2005; Vemuri, 1984).

The operating characteristics of patient waiting lines in the hospital pharmacy of OAUTHC using four service channels are presented in Table 3 . The results indicate that patients queued for about 3- 5 seconds $\left(t_{\mathrm{q}}\right)$ and stayed for about $2-6$ minutes in the system. This implies that with four dispensing pharmacists actively working, patients did not need to spend a long time in the pharmacies. However, personal observation showed that most of the time only two or three of the pharmacists attended to patients on the queues while the others were occupied 
with other activities in the pharmacies. The queuing characteristics were calculated using three or two servers and the results are presented in Table 3. With two service channels, patients spent about 6 minutes in the pharmacy while the time on the queues was up to 4 minutes. With four service channels, the mean utilisation factors $\left(P_{w=}=0.04-0.08\right)$ of the facilities were quite low; this translates to a capacity utilization of $4 \%$ to $8 \%$. On the other hand, the mean utilisation factors were highest $\left(\mathrm{P}_{\mathrm{w}}=0.08-0.66\right)$ for two service channels, that is, capacity utilisation of $13 \%$ to $66 \%$. With these utilisation factors, the number of patients on the queues was still reasonable (1 - 2 patients). The number of dispensing pharmacists could be kept at two in the outpatient units while other pharmacists are engaged in other tasks in the pharmacies. However, the dispensing duties may be rotational to avoid undue stress as a result of being stationed at routine activities for the full work hours each day.

Similarly, the operating characteristics of waiting lines at the cashiers' counters are presented in Table 4. The values recorded for the parameters as shown in row A of the table were calculated from the observed mean arrival rates $(\lambda)$ and mean service rates $(\mu)$. The results in row $\mathrm{A}$ for the hospital pharmacy indicate that patients queued for about 8 minutes before service while they spent more than 14 minutes in the paying system. This delay may be of concern considering the state of ill-health of some of these patients and the fact that they would have to join queues in some other service units of the hospitals. The mean utilisation factor of the operation is quite high $\left(\mathrm{P}_{\mathrm{w}}=0.90\right.$; capacity utilisation of up to $\left.90 \%\right)$ but the resultant long queues (up to 9 patients waiting on the queue) and the waiting time may require additional service channels. Similarly, the length of queue and the waiting time can be reduced either by increasing the service rates or making a creative design change such as modifying the service channels to improve the process.

On the other hand, using the simulation technique, rows $B$ and $C$ of Table 4 present the queuing characteristics assuming utilisation factors $\left(\mathrm{P}_{\mathrm{w}}\right)$ of 0.70 and 0.65 that is, capacity utilisation of $70 \%$ and $65 \%$ for the queuing systems. This analysis assumed a constant arrival rate at each facility but varying the service rates to obtain utilisation factors $\left(\mathrm{P}_{\mathrm{w})}\right.$ of 0.70 and 0.65 gave the operating characteristics in rows $\mathrm{B}$ and $\mathrm{C}$ of Table 4 . As the service rates increased, the performance measures of the waiting lines also improved remarkably and the number of people waiting in line was about two in the pharmacy. The probability values of no patient in the systems $\left(\mathrm{P}_{\mathrm{o}}\right)$ also increased with improved service rates.

This result agrees with the findings of a previous study which evaluated the characteristics of waiting lines in some food service systems in Nigeria (Oyebisi et al., 1999). Service rates may be increased at the payment counters in order to reduce the total patient waiting time in the pharmacy. The application of modern technology in cash transactions and accounting records may help to speed the process; for instance, the adoption of new technologies such as electronic payment and computerised recording system may relieve the delay caused by manual operations and this can facilitate a smooth flow of the dispensing process.

The results of a previous study showed that the hospital pharmacists were well disposed to the introduction of such technologies to improve some aspects of service operations in the pharmacies (Afolabi and Oyebisi, 2007b). On the other hand, there may be the need for a process redesign or adding more parallel service channels. This latter option will increase the 
number of serving personnel with the attendant increased service cost in terms of physical arrangement and personnel costs. However, it is necessary to maintain an economic balance between reasonable utilisation factors and moderate sized queues in the operating system. Essentially, new systems should be carefully assessed to be sure they contribute more to the ultimate focus of enhancing pharmacist involvement in patient care rather than just speed the process of dispensing with little time for patient counselling. Pharmacists would definitely be more satisfied at their jobs if they were able to dispense what they consider to be a 'comfortable' number of prescriptions per hour that would enable them to counsel patients properly and attend to other clinical practice contributions to patient care.

Other strategies that could significantly speed the process of service delivery in the pharmacy include the adoption of new technologies such as electronic payment and computerisation of some of the pharmacists' activities. However, the use of new technologies may not necessarily contribute to better dispensing procedure and rational use of medicines. Other areas of positive turn around in service delivery could be a job redesign and continuing reorientation of pharmacists to contemporary and emergent techniques in clinical skills and medicines management.

\section{Conclusion}

The healthcare system continues to experience dynamic change as a result of factors such as government influence, economic pressures, the biotechnology development of medicines and the continued development of robotics and automation. The pharmacy is integral to the operations of health care delivery and it should grow from a medicine distribution system to a very effective means by which patient health care may be improved through proper interpretation of the prescriptions, monitoring and follow-up of patient medicine therapy regimens. The future of pharmacy profession, as part of the healthcare system, does not lie in the dispensing or distribution of medications but in the provision of information and medicine therapy recommendations to other healthcare providers and the patients concerning rational therapeutics. As a result of these advances, the pharmacist must be prepared to meet the needs of the healthcare system and most importantly, to adopt appropriate technology in the provision of pharmaceutical services.

\section{Author details}

Margaret O. Afolabi and Omoniyi Joseph Ola-Olorun

*Address all correspondence to: bunmafol@oauife.edu.ng, bunmafol@gmail.com

Department of Clinical Pharmacy and Pharmacy Administration, Faculty of Pharmacy, Obafemi Awolowo University, Ile-Ife, Nigeria 


\section{References}

[1] Afolabi, M. O. (2005). "Evaluation of Operations Management of Pharmacies in Selected Teaching hospitals in Southwestern Nigeria", Ph.D. thesis (Unpublished). Obafemi Awolowo University, Ile-Ife, Nigeria, pp: 205-211.

[2] Afolabi, M.O. and Erhun, W.O. (2003): Patients' response to waiting time in an outpatient pharmacy in Nigeria. Tropical Journal of Pharmaceutical Research 2 (2):207-214.

[3] Afolabi, M. O. and Oyebisi, T. O. (2007a). Pharmacists' Perceptions of Barriers to Automation in Selected Hospital Pharmacies in Nigeria. Journal of Pharmacy Practice, 20: 64-71.

[4] Afolabi, M.O. and Oyebisi, T.O. (2007b). Pharmacists, Attitude to the Introduction of Automated Techniques in the Delivery of Pharmaceutical Services in Selected Nigerian Teaching Hospitals. Journal of Pharmacy Practice 20 (1), 72-81.

[5] Anderson, P. O., McGuinness, S. M. and Bourne, P. E. (2010). Pharmacy Informatics. New York: CRC Press.

[6] ASHP (2007). American Society of Health-System Pharmacists' statement on the pharmacist's role in informatics. Am J. Health-Sys. Pharm., 64: 200-203.

[7] Bozzette, S. A., D’Amato, R. D., Morton, S., Harris, A., Meili, R. and Taylor, R. (2001). Pharmaceutical Technology Assessment for Managed Care: Current Practice and Suggestions for Improvement. [Website]. Available from: <http://www.questia.com> [Accessed: 20 March, 2009].

[8] Cairns, C. (2002). Robots and Automation: a UK perspective. Hospital Prescriber, Europe Spring 02. [Website]. Available from: <http://www.hospital prescriber.com> [Accessed: 8 September, 2010].

[9] Dixon, D. R. and Dixon, B. J. (1994). Adoption of information technology enabled innovations by primary care physicians: model and questionnaire development. Proc. Annu. Symp. Comput. Appl. Med. Care, 1994, pp. 631-635.

[10] Encyclopaedia Britannica (2010). Mechanisation. Encyclopaedia Britannica Online. [Website]. Available from: <http://www.britannica.com> [Accessed: 7 April, 2010].

[11] Fitzpatrick, R., Cooke, P., Southall, C., Kauldhar, K. and Waters, P. (2005). Evaluation of an automated dispensing system in a hospital pharmacy dispensary. The Pharmaceutical Journal, 274: 763-765.

[12] Galloway, L. (2000). Principles of Operations Management $2^{\text {nd }}$ ed. Ohio: Int. Thompson Bus. Press.

[13] Garsombke, T.W. and Garsombke, D.J. (1989). Strategic implications facing small manufacturers: the linkage between robotization, computerization, automation and performance. Journal of Small Business Management, 27 
[14] Genus A, Kaplani M.(2002) Managing operations with people and technology. Intern Journ of Techn Manag.; 23(1-3):189-200

[15] Grizzle, A.J., Mahmood, M.H., Ko, Y., Murphy, J.E., Armstrong, E.P., Skrepnek, G.H. et al. (2007). Reasons provided by prescribers when overriding drug-drug interaction alerts. Am J Manag Care, 13: 573-578.

[16] Hemelink, C. J. (1997). New Information and Communication Technologies, Social Development and cultural change. United Nations Research Institute for Social Development (UNRISD). UNRISD Discussion. No. 86.

[17] Ilori, M. O. (2006). "From Science to Technology and Innovation management." An Inaugural Lecture Delivered at Oduduwa Hall, Obafemi Awolowo University, IleIfe. May 30. Inaugural Lecture Series 191. O.A.U. Press Ltd., Ile-Ife. Nigeria.

[18] Jähnke, R. W. O. (2004). Counterfeit Medicines and the GPHF-Minilab for rapid drug quality verification. PharmazeutischeIndustrie, 66 (10): 1187-1193.

[19] Jähnke, R. W. O., Kusters, G. and Fleischer, K. (2001). Low-cost quality assurance of medicines using the GPHF-Minilab ${ }^{\circledR}$. Drug Information Journal, 35: 941 - 945.

[20] Jeffcoat, M. K. (2004) Eye of newt, toe of frog. J. Am. Dent. Assoc., 135 (5): 546-547.

[21] Kawamoto, K., Houlihan, C. A., Balas, E. A. and Lobach, D. F. (2005). Improving clinical practice using clinical decision support systems: a systematic review of trials to identify features critical to success. BMJ., 330(7494): 765

[22] Kelly, W. N. (2006). Pharmacy, what it is and how it works $2^{\text {nd }}$ edition. Boca Raton: CRC Press pharmacy education series.

[23] Kling, R. (1996). Computerisation at Work in Computerisation and Controversy; Value Conflicts and Social Choices (2nd edition). San Diego, CA: Academic Press.

[24] Langabeer II, J.R. (2008). Health care operations management: a quantitative approach to business and logistics. Sudbury,MA, Jones and Barlett Pub., pp 6-7.

[25] Lin, A.C, Jang, R and Sedani D. (1996): Re-engineering a pharmacy to facilitate patient counselling. American Journal of Health-System Pharmacy 53: 1558-64.

[26] Lin, A.C., Jang, R., Lobas, N., Heaton, P., Ivey, M. and Nam, B. (1999): Identification of Factors Leading to Excessive Waiting Times in an Ambulatory Pharmacy. Hospital Pharmacy 34 (6):707-712.

[27] Local Enterprise Authority. (2009). Technology Adaptation and Adoption. [Website]. Available from: <http://www.lea.co.bw/article.php?id_mnu=48> [Accessed: 9 May, 2009].

[28] New, C (1998)'The state of operations management in the UK - a personal view', International Journal of Operations and Production Management 18(7-8):675-679

[29] Oyebisi, T.O., Ilori, M.O. and Oyeleke, T.O. (1999): Characteristics of the waiting line in selected traditional food service systems in Osun State of Nigeria. Nigeria Institute 
of Industrial Engineers (NIIE) Proceedings of 1999 Annual Conference held at Ibadan, p 74-88.

[30] Panthong S, and Daosodsai P. Work Analysis Model of Hospital Pharmacy Services at Banphai Hospital Thailand. Malaysian Journ Pharm Sci .2005; 3(2) 47

[31] Ridley, M. (2006). Genome. New York, NY: Harper Perennial.

[32] Savery, LK and Choy RM (1999) “Cutting edge' technology: an avant garde solution of operational effectiveness or careerist nightmare?", Intern Journ of Techn Manag; 17(1-2):145-156

[33] Slowiak, J. M, Huitema B. E. and Dickinson A (2008). Reduce wait time in a hospital pharmacy to promote customer satisfaction. Quality Management in Health Care.; 17 (12): 112-127.

[34] Sood, S. P., Prakash, N., Agrawal, R. K. and Foolchand, A. A. B. (2008). Telepharmacy and e-Pharmacy: Siamese or discrete? International Journal of Healthcare Technology and Management, 9 (5-6): 485-494.

[35] Thompson, T. G. and Brailer, D. J. (2006). The Decade of Health Information Technology: Delivering Consumer-centric and Information-rich health care. Framework for strategic action. [Website]. Available from: <www.nhs.gov/healthit/documents/ hitframework.pdf> [Accessed: 3 June, 2009].

[36] Toohey, J. B., Herrick, J. D., and Trautman, R. T. (1982): Adaptation of a workload measurement system. American Journal of Hospital Pharmacy 39: 999-1004.

[37] Vemuri S. (1984): Simulated analysis of patient waiting time in an outpatient pharmacy. American Journal of Hospital Pharmacy 41: 1127-30.

[38] Wild, R. (1999). Essentials of Production and Operations Management. 5th ed. Great Britain" Wiltshire: Redwood Books.

[39] Winslade, N. E., Strand, L. M., Pugsley, J. A. and Perrier, D. G. (1996). Practice Functions Necessary for the Delivery of Pharmaceutical Care. Pharmacotherapy, 16 (5): 889 -898 . 